\title{
Effects of pre-experimental knowledge on recognition memory
}

\author{
Chris M. Bird, ${ }^{1,4}$ Rachel A. Davies, ${ }^{2}$ Jamie Ward, ${ }^{3}$ and Neil Burgess ${ }^{1}$ \\ ${ }^{1}$ UCL Institute of Neurology and UCL Institute of Cognitive Neuroscience, University College London, London WC1N 3AR, \\ United Kingdom; ${ }^{2}$ University College London, London WC1E 6BT, United Kingdom; ${ }^{3}$ School of Psychology, University of Sussex, \\ Brighton BN1 9QH, United Kingdom
}

\begin{abstract}
The influence of pre-experimental autobiographical knowledge on recognition memory was investigated using as memoranda faces that were either personally known or unknown to the participant. Under a dual process theory, such knowledge boosted both recollection- and familiarity-based recognition judgements. Under an unequal variance signal detection model, pre-experimental knowledge increased both the variance and the separation of the target and foil memory strength distributions, boosting hits and correct rejections. Thus, pre-experimental knowledge has profound effects on the multiple, interacting processes that subserve recognition memory, and likely in the neural systems that underpin them.
\end{abstract}

[Supplemental material is available for this article.]

Recognition memory paradigms are commonly used to probe the neural underpinnings of declarative memory in both humans and animals (Eichenbaum et al. 2007; Squire et al. 2007). Surprisingly, the impact of pre-experimental knowledge of items on performance of recognition memory tasks has only rarely been studied. Nevertheless, this factor may have a critical influence, not only on recognition performance, but also on the brain structures that are recruited during encoding and retrieval (Bird and Burgess 2008; Trinkler et al. 2009; Poppenk et al. 2010). The present study aimed to characterize the impact of pre-experimental knowledge on recognition memory, in particular on the parameters used to fit recognition memory data according to two models based on a dual-process theory ([DPT] recollection and familiarity) and an unequal variance signal detection ([UVSD] the magnitude and variance of the memory strength signal).

Recognition memory for pre-experimentally known faces is superior to that for unfamiliar faces (Klatzky and Forrest 1984). Interestingly, patients with hippocampal damage typically perform normally on forced-choice recognition of unfamiliar faces (Bird and Burgess 2008), unless tested after a 24-h delay (Reed and Squire 1997). In an imaging study of face recognition memory, pre-experimentally known faces were found to activate the hippocampus whether they were targets or foils, whereas hippocampal activity did not differentiate the old/new status of test items (Trinkler et al. 2009). We suggested that representations of pre-experimentally known faces include associated information (mediated by the hippocampus), which boosts recognition performance. Unfamiliar faces have no pre-experimental associations (excepting look-alike coincidences) so recognition memory must rely more heavily on perceptual representations, which may not require the hippocampus.

Here, we tested face recognition memory in students from two universities, using faces from the same two universities as memoranda. Thus, half the test items were pre-experimentally known to one group of students but not to the others and vice versa. Recognition judgements were made using confidence

\section{${ }^{4}$ Corresponding author.}

E-mail chris.bird@ucl.ac.uk; fax 44-20-78132835.

Article is online at http://www.learnmem.org/cgi/doi/10.1101/lm.1952111. ratings, allowing us to assess how accuracy changes with confidence by plotting receiver operating characteristics (ROCs) and fitting these to two prominent models of recognition memory (DPT and UVSD). We also asked participants to indicate whether they "remembered" anything specific about the test items from the study phase.

We tested 22 female, second-year undergraduate psychology students (11 from Sussex University, 11 from University College London [UCL]). Stimuli were 144 photographs of students (72 from UCL, 72 from Sussex; from the same courses as the participants). Stimuli were cropped to an oval shape and presented on a white background using a computer monitor and PowerPoint (http://office.microsoft.com).

Half of the items ( 36 from UCL, 36 from Sussex) were used as targets. Targets and foils were fully counterbalanced across participants from both universities. There was a Study phase, a Test phase, and a Rating phase. In the Study phase, each of the 72 target faces was presented individually for $5 \mathrm{sec}$. Participants were required to make a male/female judgment for each face and were told that their memory for the faces would be tested. The participants were also told that while some of the faces would be known to them, their task was to attend to each face. The Test phase followed completion of a brief distractor task (Ravens Advanced Progressive Matrices Set II [Raven 1976]). Each of the 144 faces was presented individually. Participants decided whether they had seen the item in the preceding Study phase and recognition judgements were made on a six- point confidence scale, where $6=$ confident the item was previously studied and $1=$ confident the item was not previously studied. Participants were additionally asked to give a "remember" response if they retrieved a specific memory of the item from the study phase (Rotello and Zeng 2008). In the final Rating phase, participants rated all 144 faces for how well they were personally known to them on a five- point scale where 1 = "not known," $2=$ "possibly familiar," $3=$ "familiar," $4=$ "definitely familiar," and $5=$ "very familiar" (i.e., a close friend). It was explained that half of the faces were of people from a different university and therefore unlikely to be familiar.

To analyze performance, each participant's recognition judgements were used to generate two separate ROCs, one for each set of stimuli (faces from UCL or faces from Sussex). The 

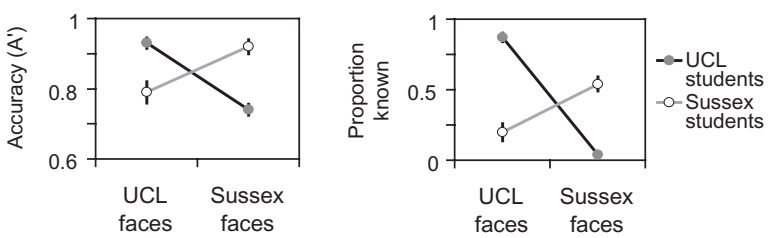

Figure 1. Accuracy data and proportions of items rated as preexperimentally known (rated 2-5; "possibly familiar" - "very familiar"). Data are split according to which university the participant was from and which university the item was from. In subsequent analyses, data from all participants were pooled and referred to as pre-experimentally known (items from the same university as the participant rated as known) and pre-experimentally unknown (all other items).

ROC shows the relationship between "yes" responses to old items and "yes" responses to new items at progressively more liberal confidence ratings by plotting the cumulative proportions of hits ( $y$-axis) vs. false alarms ( $x$-axis) for each successive confidence level. Overall performance was quantified as the area under the ROC $\left(\mathrm{A}^{\prime}\right)$.

We investigated overall performance as a function of which location the stimuli were from (faces of UCL students vs. faces of Sussex students) and the location the participant was from (UCL vs. Sussex) using a $2 \times 2$ mixed ANOVA. Results are shown in Figure 1.

The effect of pre-experimental knowledge on recognition accuracy was demonstrated by a highly significant crossover interaction effect between the locations the stimuli and participants came from $\left(F_{(1,20)}=98.1, P<0.0001\right)$, but no significant effect of participant location $\left(F_{(1,20)}<1\right)$. There was a marginally significant effect of location the stimuli were from $\left(F_{(1,20)}=4.08, P=\right.$ 0.057), due to slightly higher performance for the faces from UCL. Figure 1 also shows the proportions of faces from each university that were rated as pre-experimentally known (rated 2-5) by students at the two universities. Unsurprisingly, participants rated faces from their own university as more familiar, resulting in a significant crossover interaction between item source and participant location $\left(F_{(1,20)}=300, P<0.001\right)$. There was no effect of participant location but there was a main effect of item source, with a greater proportion of faces from UCL being rated as familiar $\left(F_{(1,20)}=52.9, P<0.001\right)$ due to the greater tendency of UCL students to rate both more UCL faces as familiar and more Sussex faces as unfamiliar. In subsequent analyses we identified "preexperimentally known" faces as those from the same university as the subject that were given a rating of 2-5 (faces from the same university as the subject but given a rating of 1 were included in the "not known" items for that subject).

This finding replicates the findings of Klatzky and Forrest (1984) and Trinkler et al. (2009) who also documented advantages in recognition memory for pre-experimentally known faces. A prominent theory of memory, DPT posits that recognition judgements are based on either recollection (the retrieval of contextual details about an encoding event), or familiarity (the feeling that an item has been experienced previously without retrieval of any additional information). Several researchers have suggested that the hippocampus is necessary for recollection but not for familiarity. Given that the hippocampus is specifically activated by the pre-experimentally known faces (Trinkler et al. 2009), and that unfamiliar face recognition memory is not hippocampal dependent (Bird and Burgess 2008), the boost in recognition memory for familiar faces might reflect a selective boost in recollection. This proposal is supported by studies that have manipulated test item familiarity by pre-exposing a subset of the items before encoding. Under these conditions, recollection appears to contribute to performance, primarily by enabling subjects to reject foils that were familiar as a result of pre-exposure (Dobbins et al. 1998). A recent study using this paradigm found that source memory (which is considered to be closely related to recollection) was more accurate for pre-exposed items (Poppenk et al. 2010).

Estimates of recollection and familiarity were first calculated using the Remember/Know procedure. "Remember" responses were made by the participant during the test whilst "know" responses were all other items rated as previously seen (i.e., given a confidence rating 4-6). Parameters were fitted to the model of Yonelinas and colleagues, which assumes that both processes are independent of each other (Yonelinas et al. 1998). Table 1 shows the total numbers of "remember" responses and the responses at each confidence level for pre-experimentally known and unknown items. Interestingly, only around 50\% of items given a " 6 " confidence response were also given a "remember" response, indicating that high-confidence familiarity-based recognition often occurs for faces. The probability that an item was recollected, as calculated from these data, was 0.35 for preexperimentally known items and 0.11 for pre-experimentally unknown items. Estimates of familiarity were 1.87 and 1.01 for pre-experimentally known vs. unknown items, respectively. Recollection and familiarity estimates were also calculated for each individual and the mean values are shown in Figure 2. Pre-experimental knowledge about the stimuli significantly boosted both recollection $(t=4.6$, d.f. $=21, P<0.001)$ and familiarity $(t=6.0$, d.f. $=21, P<0.001)$.

The group data and each participant's data were used to plot ROC curves, which were then fitted to the DPT of Yonelinas and colleagues to estimate recollection and familiarity (Yonelinas et al. 1998). The group ROCs for pre-experimentally known and unknown items are shown in Figure 2. Under DPT, the $y$-intercept reflects the probability that an item is recollected and the degree of curvature of the line reflects increasing familiarity elicited by the test items (measured by d-prime; $\mathrm{d}^{\prime}$ ). These curves in Figure 2 correspond to recollection estimates of 0.41 and 0.23 and familiarity estimates of 1.73 and 0.87 for known vs. unknown items, respectively. These estimates are similar to the mean recollection and familiarity estimates calculated separately for each participant (Fig. 2B); again pre-experimental knowledge significantly boosted both recollection $(t=3.9$, d.f. $=21, P<0.005)$ and familiarity $(t=5.3$, d.f. $=21, P<0.001)$.

Thus, when our recognition data were fitted according to DPT, pre-experimental knowledge about the items boosted both recollection- and familiarity-based recognition processes, whether calculated from the remember/know responses or ROCs (Fig. 2B). This finding is not consistent with the hypothesis that pre-experimental knowledge improves performance via a selective boost in recollection of the test items, but not familiarity. It is also at odds with studies that have used pre-exposure to introduce pre-test item familiarity (Dobbins et al. 1998; Poppenk et al. 2010). However, this experimentally induced pre-exposure is very

Table 1. Distribution of responses across confidence levels

\begin{tabular}{llrrrrrrr}
\hline & & \multicolumn{7}{c}{ Confidence level } \\
\cline { 3 - 7 } & & \multicolumn{1}{c}{$\mathbf{5}$} & $\mathbf{5}$ & $\mathbf{4}$ & $\mathbf{3}$ & $\mathbf{2}$ & $\mathbf{1}$ & \multicolumn{1}{c}{$\mathbf{R}$} \\
\hline Pre-experimentally & Targets & \multicolumn{1}{c}{380} & 63 & 40 & 26 & 37 & 22 & 202 \\
known & Foils & 21 & 16 & 35 & 50 & 96 & 308 & 6 \\
Pre-experimentally & Targets & 215 & 156 & 150 & 208 & 177 & 74 & 107 \\
unknown & Foils & 8 & 34 & 94 & 222 & 380 & 320 & 4 \\
\hline
\end{tabular}

Distribution of recognition responses summed across all participants. For the remember/know analyses, "know" responses were those items given a confidence rating of 4-6 that had not been given a "remember" response. 
A
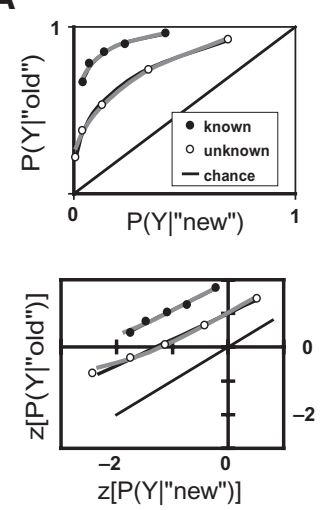

B
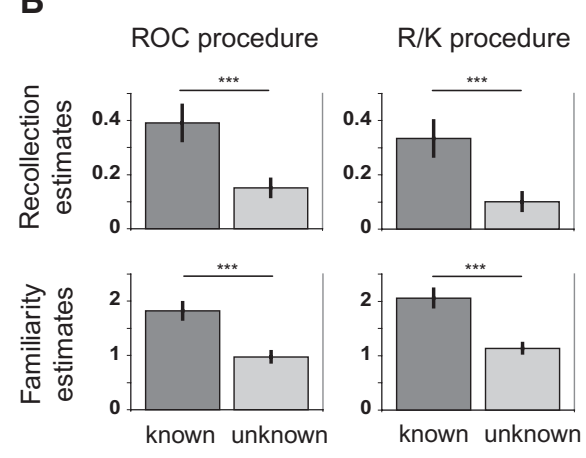

C

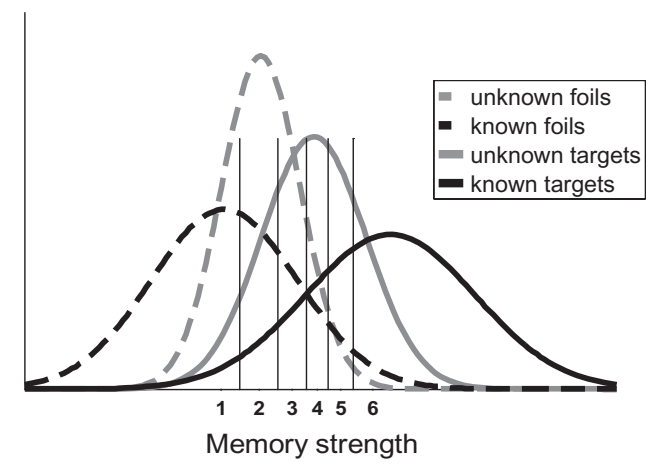

Figure 2. (A) Group averaged receiver-operating characteristics (ROCs) and z-transformed ROCs for the pre-experimentally known and unknown items. The lines between the points represent the data fitted to the dual process theory (DPT: black line) and the unequal variance signal detection model (UVSD: gray line). Goodness-of-fit statistics for both models are provided in the Supplemental material. (B) Data fitted to dual-process theory. Recollection and familiarity estimates for previously known and unknown items calculated using the ROC and Remember/Know procedures. Recollection is measured as the probability that an item is recollected, familiarity is measured as d-prime. ${ }^{* * *}, P<0.005$. (C) Data fitted to the unequal variance signal detection model. The target distributions represent the memory strength values associated with the items that were studied (either pre-experimentally known or unknown), and the foil distributions represent the memory strength of the items that were not. The unknown foil distribution is used as the baseline ( mean $=0$, standard deviation $=1$ ) and the others are shifted in both mean and standard deviation relative to it. Vertical lines indicate the boundaries between the six confidence levels (as indicated on the $x$-axis). The separation of the means of the target and foil distributions is a measure of how well participants distinguished studied from unstudied items. Note that the separation of the distributions for pre-experimentally known items is greater than for pre-experimentally unknown items and the standard deviations for the known items are greater than for unknown items.

different from our manipulation of pre-experimental knowledge. Certainly, the boost in performance afforded by pre-experimental knowledge in our study far exceeds that afforded by pre-exposure to the test items in other studies (Dobbins et al. 1998; Poppenk et al. 2010). It may be that pre-experimental knowledge boosts the "distinctiveness" of the test items in a nonspecific way that affects both recollection- and familiarity-based recognition judgements. Thus, a conclusion for dual process models is that the presence of pre-experimental knowledge of the stimuli used for both targets and foils boosts familiarity-related processes, as well as recollection-related process.

In the final series of analyses, the ROC data were analyzed according to a UVSD model of recognition memory. Under this model judgements about the prior occurrence of items are made on the basis of a single internal representation of "memory strength" (i.e., a sense of prior occurrence) (Mandler 1980; Wixted 1992). Thus, every test item elicits a memory strength signal that is used to determine whether that item is a target or foil by comparing the memory strength to a criterion value (items that elicit the strongest response will be most confidently judged as "old," while items that elicit the weakest response will be most confidently judged as "new"). The different confidence levels in the ROC correspond to different criterion values. The UVSD model allows the memory strength distributions associated with items in the studied and unstudied, pre-experimentally known and unknown groups (four distributions in total) to have different means and standard deviations. These means and standard deviations were estimated from the ROC data (using software from http://www.unifr.ch/psycho/site/units/allpsy/team/Macho). The foils of pre-experimentally unknown faces were set to have a mean of 0 and a standard deviation of 1 , so that all other distributions were scaled relative to the foils of pre-experimentally unknown faces. Note that this method of fitting the data assumes that all recognition judgements (for pre-experimentally known and unknown items) are made using the same memory strength axis and the same confidence level boundaries. An alternative would be to assume that participants were aware that some items were pre-experimentally known whereas others were not, and different confidence criteria were used for the two item classes. The data were also analyzed according to this model and these results are presented in the Supplemental material, together with a brief justification for the analysis we have used in the main paper.

The results are shown in Figure $2 \mathrm{C}$ and Table 2 . Of note, the standard deviation of the target distributions is always larger than the standard deviation of the respective foil distribution (known targets $>$ known foils, unknown targets $>$ unknown foils). This common finding (e.g., Glanzer et al. 1999) is usually explained by fluctuating levels of attention paid to the targets at encoding; well-attended items receive a larger boost in memory strength than less well-attended items (Wixted 2007). Pre-experimental knowledge also increases the separation of the memory strength distributions for targets and foils, which makes these items more discriminable and hence boosts performance.

Interestingly, both the target and foil distributions for pre-experimentally known items are shifted relative to the target and foil distributions for pre-experimentally unknown faces. Thus, not only are targets more confidently identified as "old,"

Table 2. UVSD model: Accuracy and variance

\begin{tabular}{lll}
\hline & Memory strength & Standard deviation \\
\hline $\begin{array}{c}\text { Pre-experimentally } \\
\text { known targets }\end{array}$ & $3.30(2.92-3.66)$ & $2.16(1.84-2.48)$ \\
$\begin{array}{c}\text { Pre-experimentally } \\
\text { unknown targets }\end{array}$ & $1.35(1.22-1.48)$ & $1.32(1.20-1.44)$ \\
$\begin{array}{c}\text { Pre-experimentally } \\
\text { known foils }\end{array}$ & $-0.93(-1.17--0.69)$ & $1.86(1.62-2.10)$ \\
$\begin{array}{c}\text { Pre-experimentally } \\
\text { unknown foils }\end{array}$ & 0 & 1 \\
\hline
\end{tabular}

ROC data fitted to the UVSD model. The data are fitted to four Gaussian distributions that vary in mean memory strength and standard deviation (see Fig. 2B). The distribution for pre-experimentally unknown foils is set to have a mean of 0 and a standard deviation of 1 and the other distributions are calculated relative to this baseline. $95 \%$ confidence intervals for the parameter estimates are shown in parentheses. 
but also foils are more confidently identified as "foils." Presumably, participants are able to use something like a "recall-to-reject" strategy; foils of pre-experimentally known faces might be correctly rejected on the grounds that they would have been remembered had they actually appeared on the list (see Brown et al. 1977).

Under the assumption that participants use the same response criteria for making their confidence judgements for preexperimentally known and unknown items, the memory strength distributions for known targets and foils have greater variance than the equivalent distributions for the unknown items (see Fig. 2C; Table 2). Consequently, the positive tail of the distribution of memory strengths for known foils exceeds that for unknown foils. This can be seen in the frequencies of responses (Table 2), where the percentage of high confidence false alarms (rating " 6 ") for the pre-experimentally known foils is higher than for the unknown foils ( $4.0 \%$ vs. $0.8 \%)$. In these instances it appears that pre-experimental knowledge of the item results in a strong feeling that the item was studied when it was not; an interesting case of proactive interference (similar to when semantically related foils are sometimes erroneously recalled or recognized with high confidence) (Roediger and McDermott 1995). This effect argues against the suggestion that pre-experimental knowledge boosts performance simply by increasing the distinctiveness of items; it is unclear why a subset of "distinct" foils would induce more high confidence false alarms. Note however, that if different decision criteria were used for pre-experimentally known vs. unknown items then direct comparisons between the variance of the distributions and the proportion of the " 6 " responses are unwarranted (see Supplemental material).

In summary: Pre-experimental knowledge substantially boosts recollection and familiarity in a recognition memory test. It is well established that known faces are processed differently from unknown faces (Ellis et al. 1979; Megreya and Burton 2006). For example, pre-experimentally known faces can be represented as unique individuals, already associated with information about their identity and particular contextual details. The richer representations for known items enable subjects to make more accurate and higher confidence recognition judgements, but may also induce high confidence false alarms in a subset of items. There was no evidence for a selective boost in recollection, which might have been expected if subjects needed to explicitly recall the encoding phase in order to differentiate pre-experimentally known targets and lures. Under a UVSD model, pre-experimental knowledge results in greater separation of the target and foil memory strength distributions. The varying amounts of knowledge we have about individuals known to us can account for the greater variance in memory strength associated with pre-experimentally known faces compared with unknown faces, which are likely to be represented more in terms of their perceptual features. The degree of pre-experimental knowledge with test items is therefore of key importance when investigating recognition memory, especially when comparing data across studies and across species, since it is likely to impact strongly on the processes by which recognition judgments are made and also on the brain regions recruited to perform the tasks.

\section{Acknowledgments}

This work was funded by the UK Medical Research Council. We thank Brad Duchaine, John Wixted, Ken Norman, and Andrew Yonelinas for helpful discussions.

\section{References}

Bird CM, Burgess N. 2008. The hippocampus supports recognition memory for familiar words but not unfamiliar faces. Curr Biol 18: 1932-1936.

Brown J, Lewis VJ, Monk AF. 1977. Memorability, word-frequency and negative recognition. QJ Exp Psychol 29: 461-473.

Dobbins IG, Kroll NEA, Yonelinas AP, Liu Q. 1998. Distinctiveness in recognition and free recall: The role of recollection in the rejection of the familiar. J Mem Lang 38: 381-400.

Eichenbaum H, Yonelinas AP, Ranganath C. 2007. The medial temporal lobe and recognition memory. Annu Rev Neurosci 30: 123-152.

Ellis HD, Shepherd JW, Davies GM. 1979. Identification of familiar and unfamiliar faces from internal and external features: Some implications for theories of face recognition. Perception 8: 431-439.

Glanzer M, Kim K, Hilford A, Adams JK. 1999. Slope of the receiver-operating characteristic in recognition memory. J Exp Psychol Learn Mem Cogn 25: 500-513.

Klatzky RL, Forrest FH. 1984. Recognizing familiar and unfamiliar faces. Mem Cognit 12: 60-70.

Mandler G. 1980. Recognizing — the judgment of previous occurrence. Psychol Rev 87: 252-271.

Megreya AM, Burton AM. 2006. Unfamiliar faces are not faces: Evidence from a matching task. Mem Cognit 34: 865-876.

Poppenk J, McIntosh AR, Craik FI, Moscovitch M. 2010. Past experience modulates the neural mechanisms of episodic memory formation. $J$ Neurosci 30: 4707-4716.

Raven JC. 1976. Advanced progressive matrices. Oxford Psychologists Press, Oxford, UK.

Reed JM, Squire LR. 1997. Impaired recognition memory in patients with lesions limited to the hippocampal formation. Behav Neurosci 111: $667-675$.

Roediger HL, McDermott KB. 1995. Creating false memoriesremembering words not presented in lists. J Exp Psychol Learn Mem Cogn 21: $803-814$.

Rotello CM, Zeng M. 2008. Analysis of RT distributions in the remember-know paradigm. Psychon Bull Rev 15: 825-832.

Squire LR, Wixted JT, Clark RE. 2007. Recognition memory and the medial temporal lobe: A new perspective. Nat Rev Neurosci 8: 872-883.

Trinkler I, King JA, Doeller CF, Rugg MD, Burgess N. 2009. Neural bases of autobiographical support for episodic recollection of faces. Hippocampus 19: 718-730.

Wixted JT. 1992. Subjective memorability and the mirror effect. J Exp Psychol Learn Mem Cogn 18: 681-690.

Wixted JT. 2007. Dual-process theory and signal-detection theory of recognition memory. Psychol Rev 114: 152-176.

Yonelinas AP, Kroll NE, Dobbins I, Lazzara M, Knight RT. 1998. Recollection and familiarity deficits in amnesia: Convergence of remember-know, process dissociation, and receiver operating characteristic data. Neuropsychology 12: 323-339.

Received July 22, 2010; accepted in revised form October 10, 2010. 


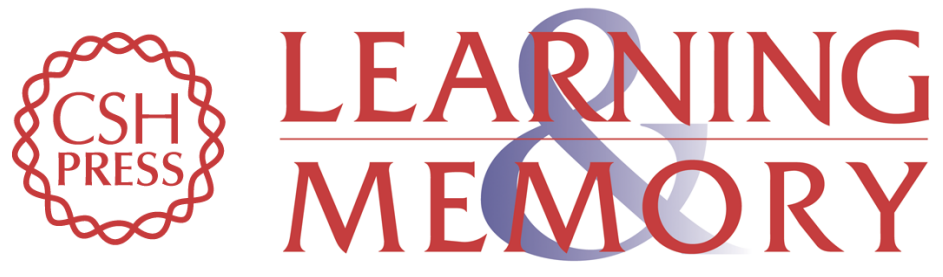

\section{Effects of pre-experimental knowledge on recognition memory}

Chris M. Bird, Rachel A. Davies, Jamie Ward, et al.

Learn. Mem. 2011, 18:

Access the most recent version at doi:10.1101/lm.1952111

Supplemental http://learnmem.cshlp.org/content/suppl/2010/12/22/18.1.11.DC1

Material

References This article cites 18 articles, 1 of which can be accessed free at:

http://learnmem.cshlp.org/content/18/1/11.full.html\#ref-list-1

License

Email Alerting Receive free email alerts when new articles cite this article - sign up in the box at the Service top right corner of the article or click here. 